\title{
A Novel Contrast for DTI Visualization for Thalamus Delineation
}

\author{
Xian Fan ${ }^{\mathrm{a}}$, Meredith Thompson ${ }^{\mathrm{a}, \mathrm{b}}$, John A. Bogovic ${ }^{\mathrm{a}}$, Pierre-Louis Bazin ${ }^{\mathrm{c}}$, Jerry L. Prince ${ }^{\mathrm{a}, \mathrm{c}}$ \\ ${ }^{\mathrm{a} J o h n s ~ H o p k i n s ~ U n i v e r s i t y, ~ B a l t i m o r e, ~ M D, ~}{ }^{\mathrm{b}}$ North Carolina State University, Raleigh, NC, ${ }^{\mathrm{J}}$ Johns Hopkins University \\ School of Medicine, Baltimore, MD
}

\begin{abstract}
It has been recently shown that thalamic nuclei can be automatically segmented using diffusion tensor images (DTI) under the assumption that principal fiber orientation is similar within a given nucleus and distinct between adjacent nuclei. Validation of these methods, however, is challenging because manual delineation is hard to carry out due to the lack of images showing contrast between the nuclei. In this paper, we present a novel gray-scale contrast for DTI visualization that accentuates voxels in which the orientations of the principal eigenvectors are changing, thus providing an edge map for the delineation of some thalamic nuclei. The method uses the principal fiber orientation computed from the diffusion tensors computed at each voxel. The three-dimensional orientations of the principal eigenvectors are represented as five dimensional vectors and the spatial gradient (matrix) of these vectors provide information about spatial changes in tensor orientation. In particular, an edge map is created by computing the Frobenius norm of this gradient matrix. We show that this process reveals distinct edges between large nuclei in the thalamus, thereby making manual delineation of the thalamic nuclei possible. We briefly describe a protocol for the manual delineation of thalamic nuclei based on this edge map used in conjunction with a registered T1-weighted MR image, and present a preliminary multi-rater evaluation of the volumes of thalamic nuclei in several subjects.
\end{abstract}

Keywords: thalamus parcellation, diffusion tensor imaging, manual delineation, orientation edge map

\section{INTRODUCTION}

The thalamus is a central relay station for neuronal communication in the brain [see Fig. 1(a)]. Distinct white matter tracts pass through specific thalamic nuclei and project to specific parts of the cerebral cortex, yielding nuclei with uniquely oriented diffusion characteristics [see Fig. 1(b)]. Parcellation of the thalamic nuclei has been recently explored using diffusion tensor imaging (DTI) $[3,4,7]$. These methods assume that the orientations of the principal eigenvectors within a given nucleus are similar, but they are distinct between different nuclei [3]. Although these papers provide

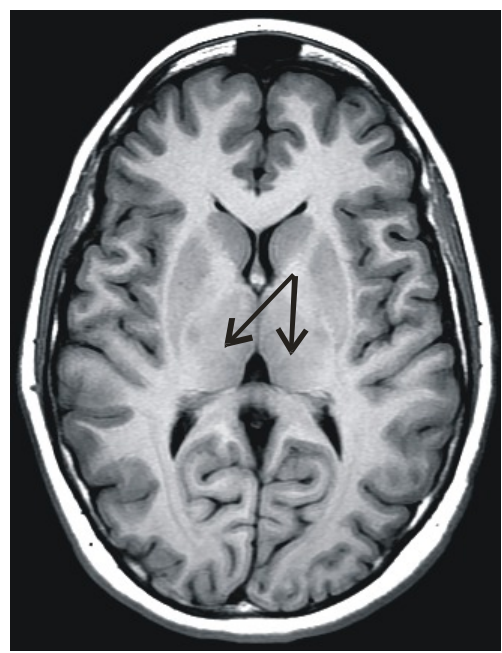

(a)

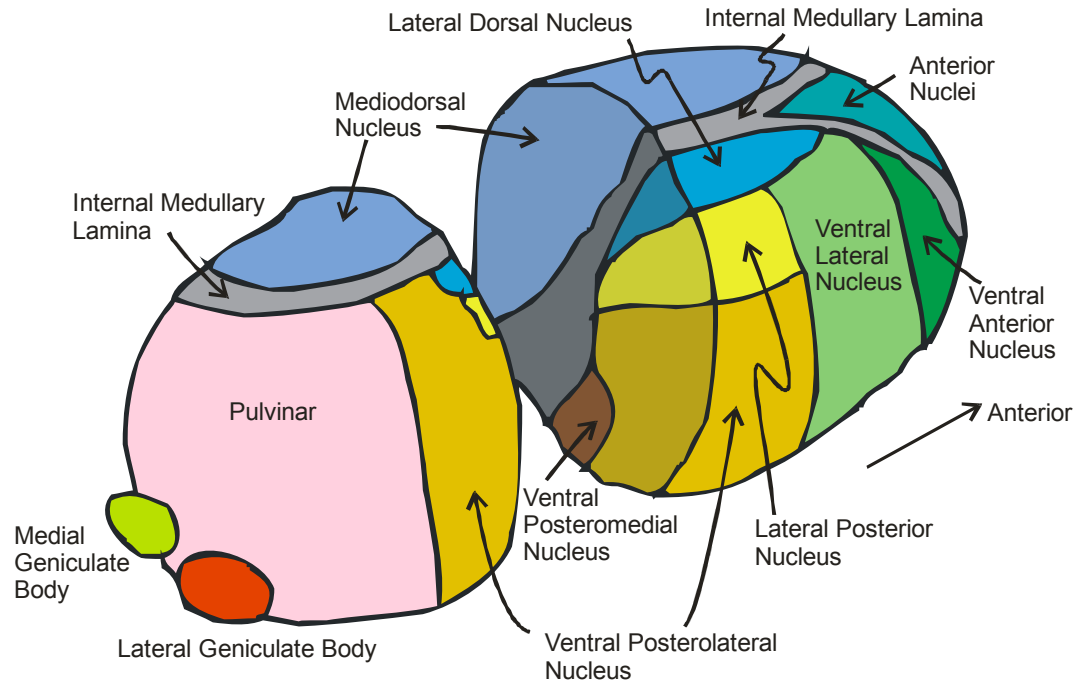

(b)

Fig. 1. (a) Location of the thalamus in the brain. (b) The thalamic nuclei. 
qualitative validation of the segmentation result, they have to date not provided any quantitative validations. This is mainly due to the difficulty in visualizing the fiber orientation, a key requirement for manual delineation, which would ordinarily provide a truth model against which to compare the automated algorithms.

Visualization of orientation in DTI has been explored in the past. In [1,2], principal eigenvectors extracted from diffusion tensors are displayed using a color map with green indicating the anterior-posterior orientation, red indicating the left-right orientation, and blue indicating the superior-inferior orientation, as illustrated in Fig. 2. While this display can give some indication of a vector's orientation, it cannot show the orientation of the whole space uniquely. In fact, since the colors are symmetric in the $x, y$, and $z$ axes, this color map can only show $1 / 4$ of the three-dimensional (3D) space uniquely, as one color may represent four different orientations or eight directions. We note that a vector's direction includes information about its "root" and "arrow" while its orientation does not.

It is important to understand that we are concerned with orientation, not direction, in this work since the principal eigenvectors can be arbitrarily multiplied by -1 when carrying out eigenanalysis of the diffusion tensor. The main goals of this paper are 1) to define an edge map defining the voxels over which the orientations are changing and 2) to specify a thalamic nuclei delineation strategy that uses the available information in both T1-weighted images and diffusion weighted images, including the new edge map.

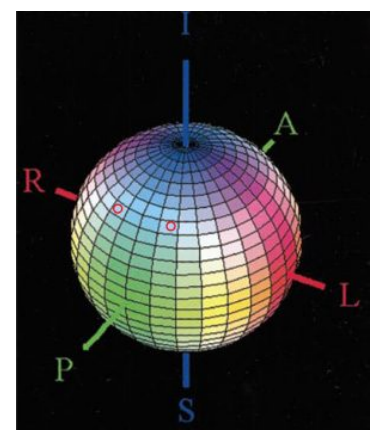

Figure 2. Illustration of the color sphere. The little red circles have the same color but depict vectors having different orientations.

\section{METHODS}

\subsection{Diffusion Tensors}

Diffusion magnetic resonance imaging acquires images that depend on the strength and direction of water diffusion in the tissues. Diffusion-weighted magnetic resonance (DW-MR) images are acquired using a pulsed gradient spin echo pulse sequence which includes control over the so-called $\mathrm{b}$-value $b$ and gradient direction $\boldsymbol{g}$. For Gaussian anisotropic diffusion the signal $S(b, g)$ at each voxel is an attenuated version of the signal $S_{0}$ that would be recorded in the absence of diffusion weighting. Specifically, the Stejskal-Tanner equation specifies the following relationship

$$
S(b, \boldsymbol{g})=S_{0} e^{-b \boldsymbol{g}^{T} D \boldsymbol{g}}
$$

where $D$ is the symmetric $3 \times 3$ diffusion tensor, which can be written as

$$
D=\left[\begin{array}{ccc}
D_{x x} & D_{x y} & D_{x z} \\
D_{x y} & D_{y y} & D_{y z} \\
D_{x z} & D_{y z} & D_{z z}
\end{array}\right]=\left[\begin{array}{lll}
\boldsymbol{u}_{1} & \boldsymbol{u}_{2} & \boldsymbol{u}_{3}
\end{array}\right]\left[\begin{array}{ccc}
\lambda_{1} & 0 & 0 \\
0 & \lambda_{2} & 0 \\
0 & 0 & \lambda_{3}
\end{array}\right]\left[\begin{array}{lll}
\boldsymbol{u}_{1} & \boldsymbol{u}_{2} & \boldsymbol{u}_{3}
\end{array}\right]^{T} .
$$

The eigen-decomposition shown in (2) yields diffusivities $\lambda_{1}, \lambda_{2}$, and $\lambda_{3}$ and orientations, $\boldsymbol{u}_{1}, \boldsymbol{u}_{2}$, and $\boldsymbol{u}_{3}$. Since the most diffusive direction corresponds to the orientation associated with the largest diffusivity, the principal eigenvalue (PEV) $\boldsymbol{u}_{1}$ is associated with the direction of maximum diffusion and is generally thought to correspond to fiber direction and often used exclusively in tractography procedures.

It is useful to distinguish gray matter from white matter by assessing the overall level of anisotropy of diffusion in a voxel. This is done by computing the fractional anisotropy (FA) according to

$$
\mathrm{FA}=\sqrt{\frac{3}{2}} \sqrt{\frac{\left(\lambda_{1}-\bar{\lambda}\right)^{2}+\left(\lambda_{2}-\bar{\lambda}\right)^{2}+\left(\lambda_{3}-\bar{\lambda}\right)^{2}}{\lambda_{1}^{2}+\lambda_{2}^{2}+\lambda_{3}^{2}}}
$$

where $\bar{\lambda}=\left(\lambda_{1}+\lambda_{2}+\lambda_{3}\right) / 3$. The FA is between zero and one and closer to one when the diffusion is more anisotropic. 


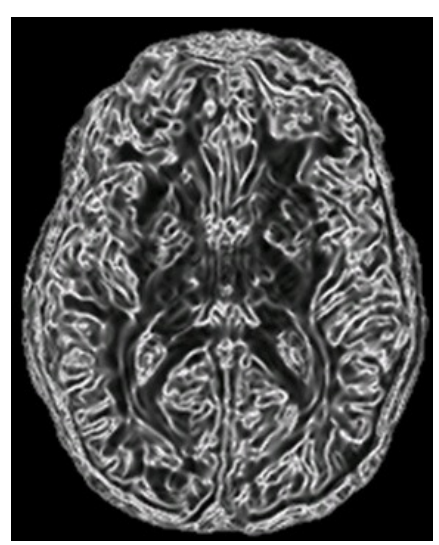

Fig. 3. An orientation edge map is the Frobenius norm of the orientation spatial gradient matrix.

\subsection{Edge Map}

Our goal is to reveal or highlight voxels within the thalamus that are on the boundary between thalamic nuclei. Since each nucleus is known to have white matter fibers that are primarily oriented in same direction and the orientations of the fibers within different nuclei are different, our strategy is to find an edge map that is large at voxels where the orientation of the principal eigenvector of diffusion is changing rapidly in one or more spatial directions.

The first step in our approach is to remove the directional ambiguity by embedding the principal eigenvectors in 5D using the Knutsson mapping [5,6]. Suppose the principal eigenvector of a voxel is $\boldsymbol{u}=\left(u_{1}, u_{2}, u_{3}\right)$; then the mapped 5D vector is

$$
M_{r 3 D}(\boldsymbol{u})=\left(v_{1}, v_{2}, v_{3}, v_{4}, v_{5}\right)=\frac{1}{\|u\|}\left(u_{1}^{2}-u_{2}^{2}, 2 u_{1} u_{2}, 2 u_{1} u_{3}, 2 u_{2} u_{3}, \frac{1}{\sqrt{3}}\left(2 u_{3}^{2}-u_{1}^{2}-u_{2}^{2}\right)\right)
$$

Only the orientation information is preserved in this transformation-both length and direction are obliterated. In particular, two vectors pointing in opposite directions in $3 \mathrm{D}$ correspond to the same $5 \mathrm{D}$ vector regardless of their relative lengths. The Knutsson mapping from the 3D unit sphere to the unit 5D hypersphere is continuous and preserves distances between vectors on the sphere. To compute the edge map of the 3D orientations in 5D space, we first apply a finite difference gradient operator on the 3D spatial grid to obtain an estimate of the gradient matrix:

$$
G=\left[\begin{array}{ccc}
\frac{\partial v_{1}}{\partial x} & \frac{\partial v_{1}}{\partial y} & \frac{\partial v_{1}}{\partial z} \\
\frac{\partial v_{2}}{\partial x} & \frac{\partial v_{2}}{\partial y} & \frac{\partial v_{2}}{\partial z} \\
\vdots & \vdots & \vdots \\
\frac{\partial v_{5}}{\partial x} & \frac{\partial v_{5}}{\partial y} & \frac{\partial v_{5}}{\partial z}
\end{array}\right]
$$

Since the gradient matrix contains all the edge information from different directions, the Frobenius norm can be used to characterize the whole edge map:

$$
\|G\|_{F}=\sqrt{\sum_{j=1}^{3} \sum_{i=1}^{5} G_{i j}^{2}}
$$

An edge map depicting $\|G\|_{F}$ is shown in Fig. 3. 


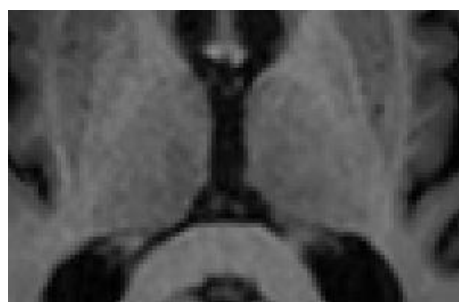

(a)

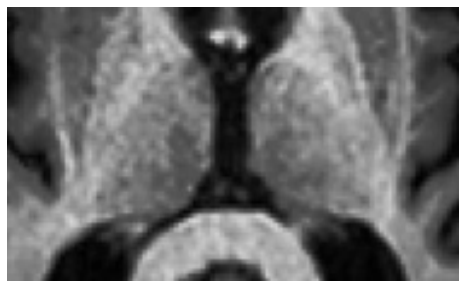

(c)

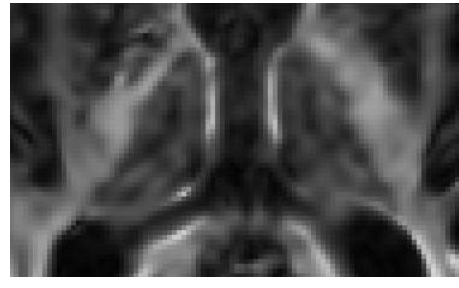

(b)

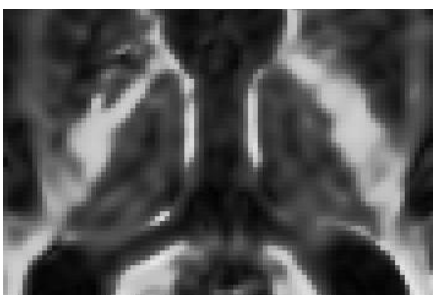

(d)

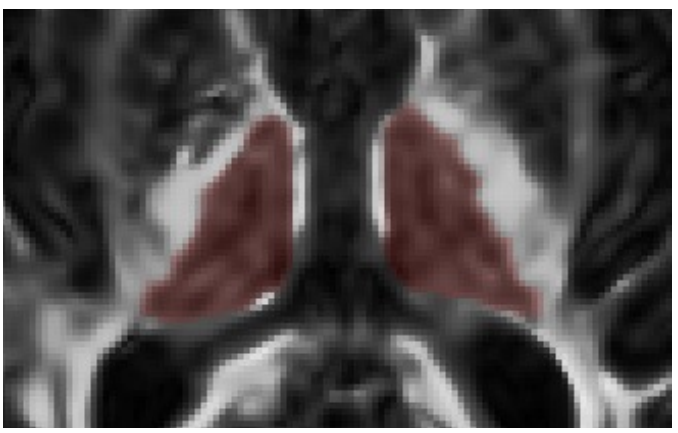

Fig. 5. Delineated thalamus shown on enhanced FA image in the axial orientation.

Fig. 4. (a) T1-weighted MP-RAGE and (b) FA images. Histogram enhanced (c) T1-weighted and (d) FA images.

\section{RESULTS:}

\subsection{Thalamic Parcellation}

The main purpose of the edge map of principal eigenvector orientation in the present study is to provide visual information to human raters so that they can delineate the thalamic nuclei manually. A comprehensive 48-page protocol was developed for manual delineation of thalamic nuclei using coregistered DW-MRI ("Jones-30" protocol, $b=$ $1000 \mathrm{~s} / \mathrm{mm}^{2}$ ) and T1-weighted (MP-RAGE) MRI data. This section provides a brief overview of the protocol, with specific emphasis on how the orientation edge map provided key information in the delineation. The MIPAV (Medical Image Processing, Analysis, and Visualization) software (NIH, Bethesda MD) was used for all image manipulation and labeling.

First, the thalamus itself was labeled using the T1-weighted (T1w) image. Both the FA and the T1w images [see Figs. 4(a) and (b)] are used to delineate the thalamus taking care to avoid including nearby structures such as the stria medullaris and the internal capsule. Histogram emphasis is applied to both the T1w and FA images within an ROI containing the thalamus, as shown in Figs. 4(c) and (d). The orientation edge map was not used in this initial step. Axial slices are labeled until the thalamus becomes unclear at the top and bottom, at which time sagittal and coronal images are used to complete the delineation. A typical result is shown in Fig. 5. Scrolling through a tri-planar view, as shown in Fig. 6, should be carried out to verify that the whole of the thalamus is captured and that no extra structures are included. This process takes about 4 hours.

The inferior thalamic nuclei-i.e., the lateral geniculate nucleus and the medial geniculate nucleus - are labeled next. These can be identified on the T1w images; a typical result is shown in Fig. 7. This process takes about 1 hour. All delineations should be verified on a tri-planar view for smoothness and continuity.

The next step is to delineate the major nuclear groups of the thalamus: 1) the medial group comprising the mediodorsal nucleus alone; 2) the anterior group comprising the anterior nucleus alone; and the 3) lateral group comprising the lateral dorsal nucleus, the ventral lateral nucleus, the ventral anterior nucleus, the lateral posterior nucleus, the ventral posterolateral nucleus, and the pulvinar. It can be seen from Fig. 1(b) that the reason that these nuclei might be grouped in this way is because of the presence of the internal medullary lamina, which is approximately a sagitally-oriented sheet running roughly posterior to anterior with a split on the anterior end. It turns out that the orientation edge map reveals the internal medullary lamina quite nicely and therefore can be used to delineate these structures. 


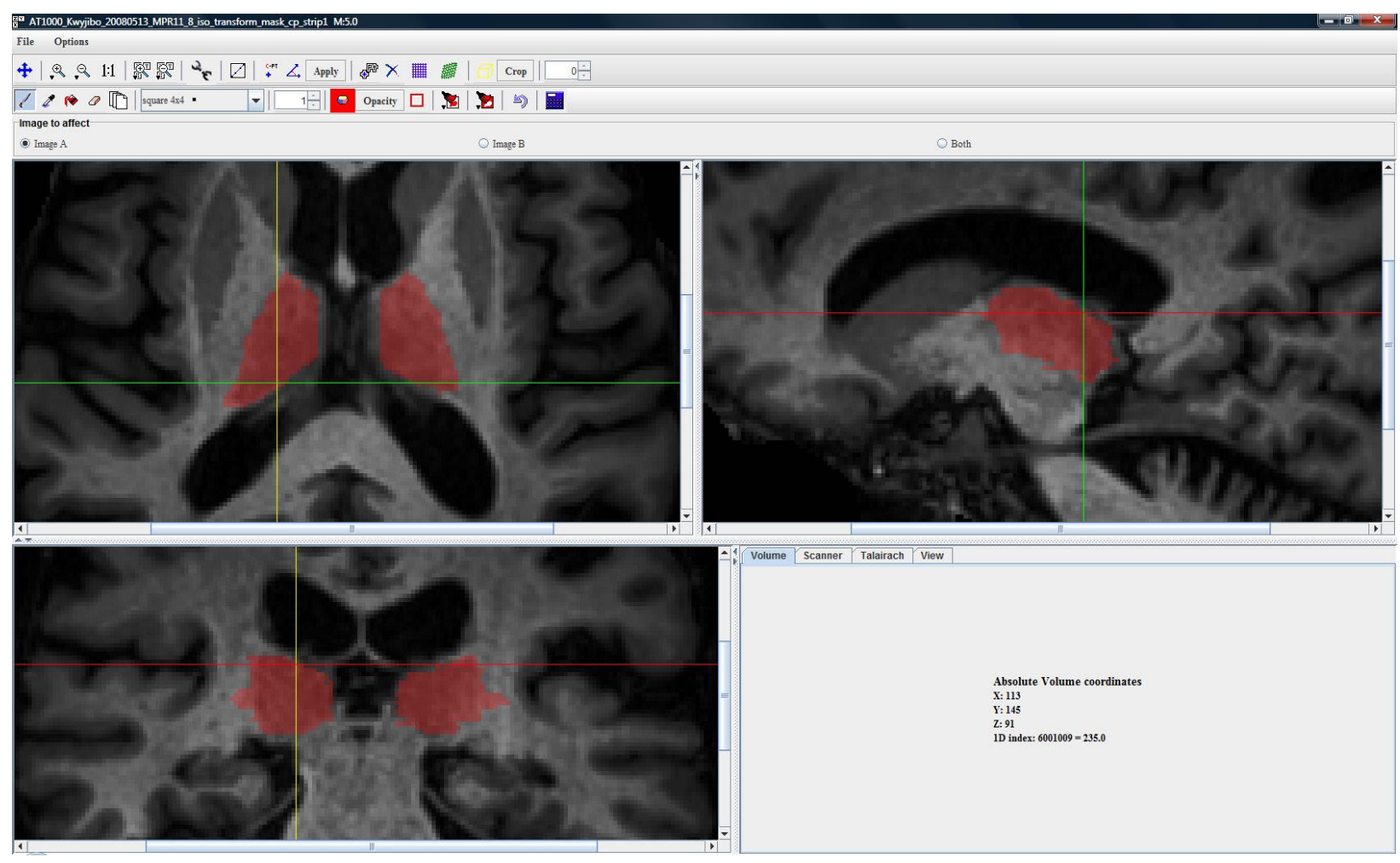

Fig. 6. Delineated thalamus shown on a triplanar view.

The medial nucleus is delineated using the edge map predominantly in the axial view, as shown in Fig. 8(a). The anterior nucleus is delineated using the edge map predominantly in the coronal view, as shown in Fig. 8(b). The lateral group is everything remaining in the thalamus that has not been painted, as shown in Fig. 8(c). This part of the delineation process takes about 2 hours.

The final stage of delineation is to identify the pulvinar which is contained within the lateral group. It is best viewed using the orientation edge map in the axial and sagittal views. Fig. 8(d) shows the pulvinar (purple) as well as the other groups that are visible in this cross section superposed on an orientation edge map.

\subsection{Comparison to Atlas}

It is informative to examine the relationship between the new edge

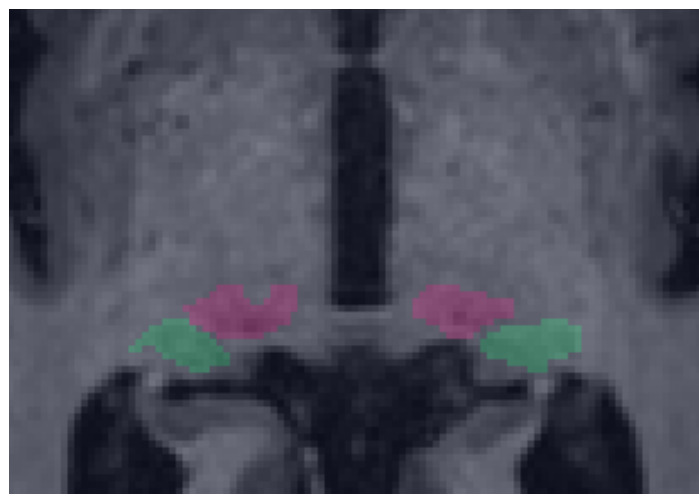

Fig. 7. Medial and lateral geniculate labeled on an axial T1w image.

map and a histological atlas [9]. Fig. 9(a) shows a close-up of an axial slice of an edge map of a thalamus. Fig. 9(b) shows the same slice with the labels overlaid. Fig. 9(c) shows the labels alone and Fig. 9(d) shows a roughly corresponding slice from an atlas. By comparison, we observe that the thalamic groups that we delineated are in good correspondence to their expected anatomical positions. Note that the lateral nuclear group (blue region on our delineation) corresponds to the labels VA, VL, and VPL in the atlas. Although not validated strictly using histological verification on the same thalamus, this illustration shows that the edges evident on the new edge map are likely to correspond to the internal medullary lamina, which is the thalamic structure separating these nuclear groups. 


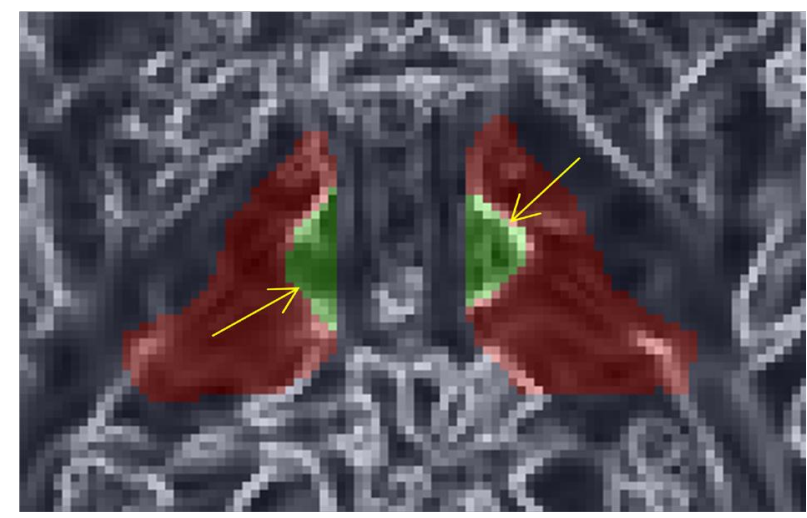

(a)

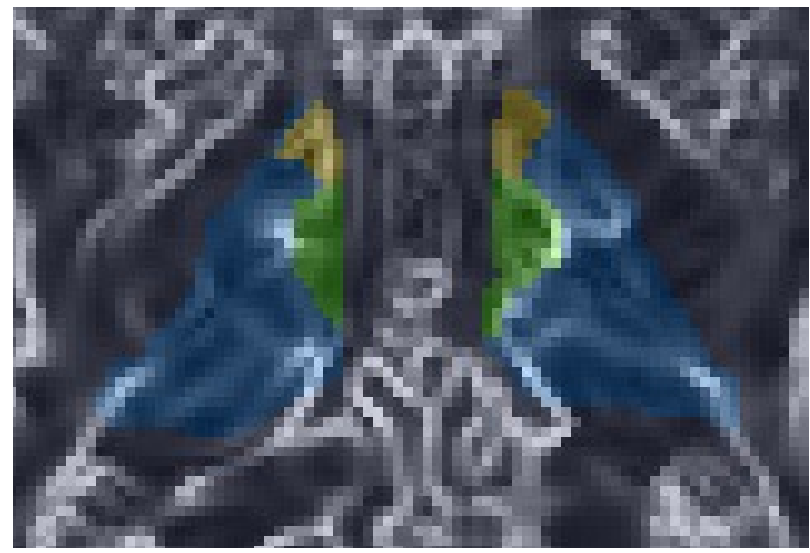

(c)

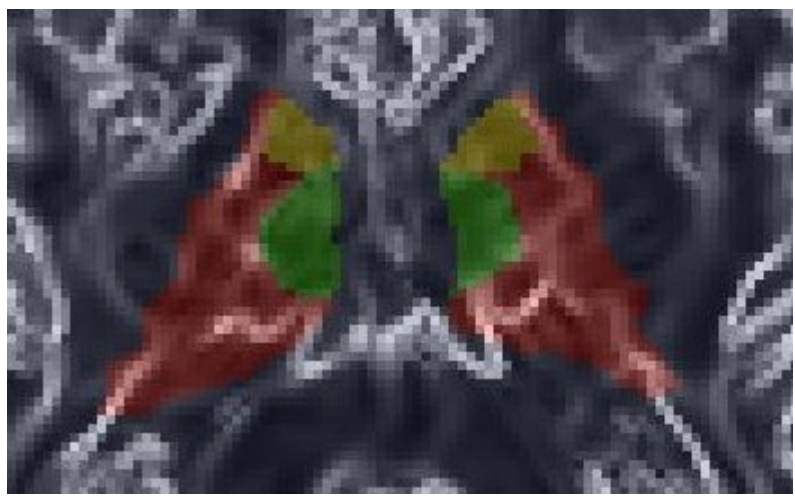

(b)

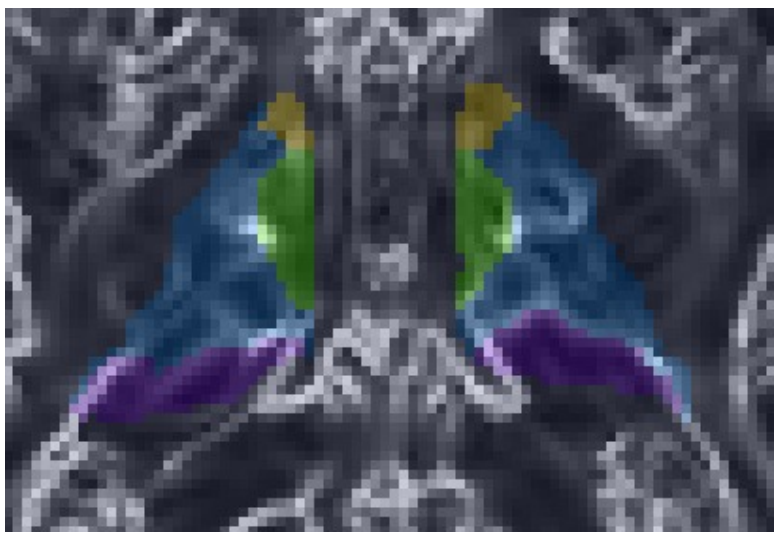

(d)

Fig. 8. (a) The medial nucleus on an edge map in the axial view. (b) The anterior nucleus (brown) on an axial edge map view. (c) The lateral nuclear group (blue) on an axial edge map view. (d) All the nuclei and nuclear groups including the pulvinar (purple).

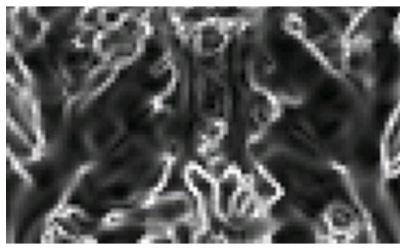

(a)

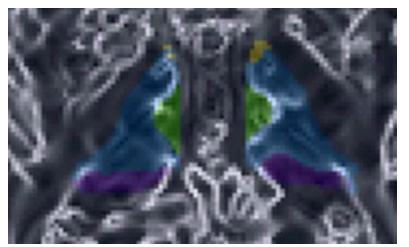

(b)

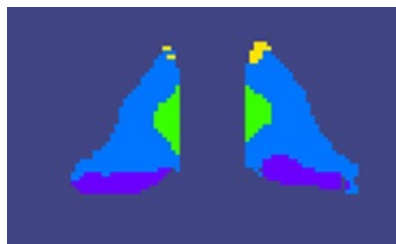

(c)

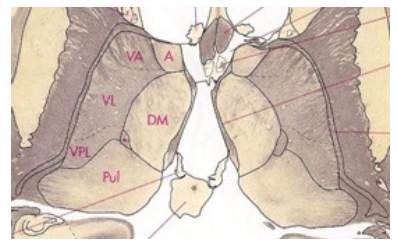

(d)

Fig. 9. (a) Close up of axial view of the orientation edge map with (b) labels overlaid. (c) The labels in isolation and (d) a comparable atlas view of a thalamus histology with labels. 


\subsection{Conventional Color Map}

Delineation of white matter fibers in the brain is often carried out by manual delineation of regions identified using a conventional DTI color map. Fig. 10 reveals why this is not likely to work in the thalamus. Fig. 10(a) shows a conventional color map and a region of interest around the medial nucleus. The boundary is clearly delineated in Fig. 10(b), which shows the orientation edge map, but is not evident at all in Fig. 10(a). In fact, several "false color edges" are evident in the conventional DTI color map, which is a problem common to all images shown using a color map rather a gray scale. Fig. 10(c) shows the correct labels on the orientation edge map. The edge of the right dorsal medial nucleus in the red box is a good example to illustrate both arbitrariness and vagueness of the conventional color map shown in Fig. 10(a).

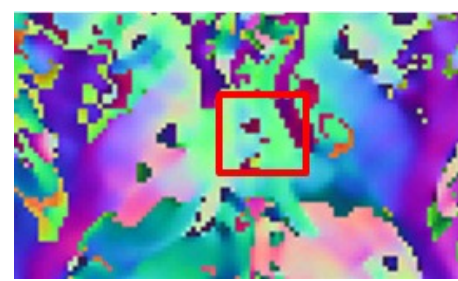

(a)

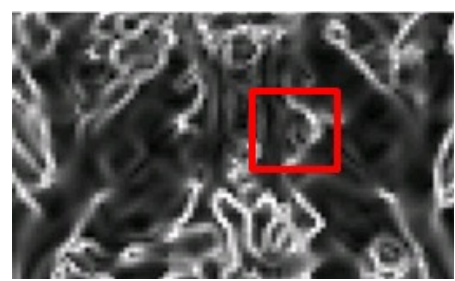

(b)

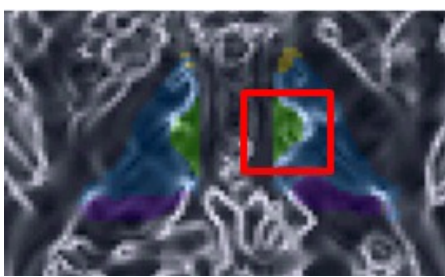

(c)

Fig. 10. (a) A thalamus displayed using a conventional color map. (b) The same thalamus depicted using the orientation edge map and (c) with superposed manual labels.

\subsection{Rater Consistency}

A preliminary analysis of rater consistency was carried out by having 4 raters delineate one thalamus. Fig. 11 shows a $3 \mathrm{D}$ rendering of one result and average Dice coefficients calculated between each rater, where Dice coefficient is defined by

$$
\mathrm{DC}=\frac{2 \operatorname{Vol}(X \cap Y)}{\operatorname{Vol}(X)+\operatorname{Vol}(Y)}
$$

where $\mathrm{n}$ is set intersection. Typically, Dice coefficients are larger for the largest volumes within a delineation, so we would expect a high Dice coefficient for the pulvinar, which is not the case. As it turns out, one rater chose a very different boundary for the anterior delineation of the pulvinar, as shown in Fig. 12. Fig. 13 gives box plots of the volumes of each nuclear group as determined by the four raters. The larger quartile in the pulvinar volume reflects the different boundary choice made by one rater.

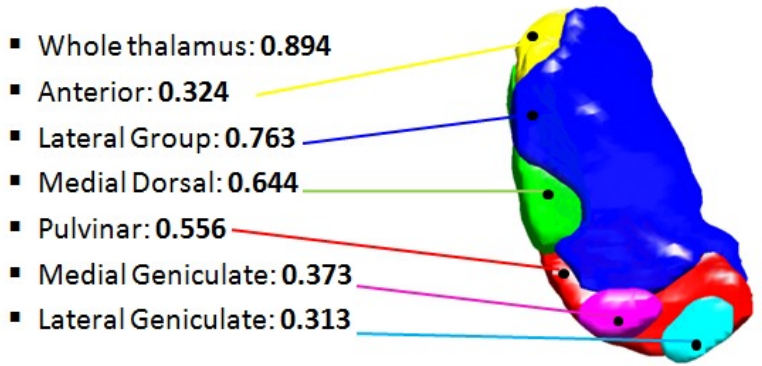

Fig. 11. Average dice coefficients between 4 raters delineating a single thalamus.

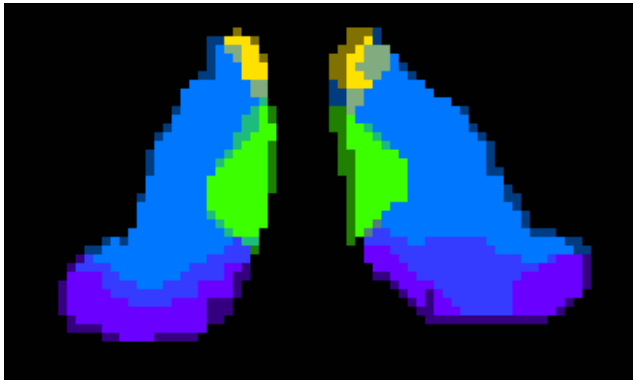

Fig. 12. Overlay of two raters' delineations. The bottom right shows very different choices for delineation of the anterior boundary of the pulvinar in this case. 


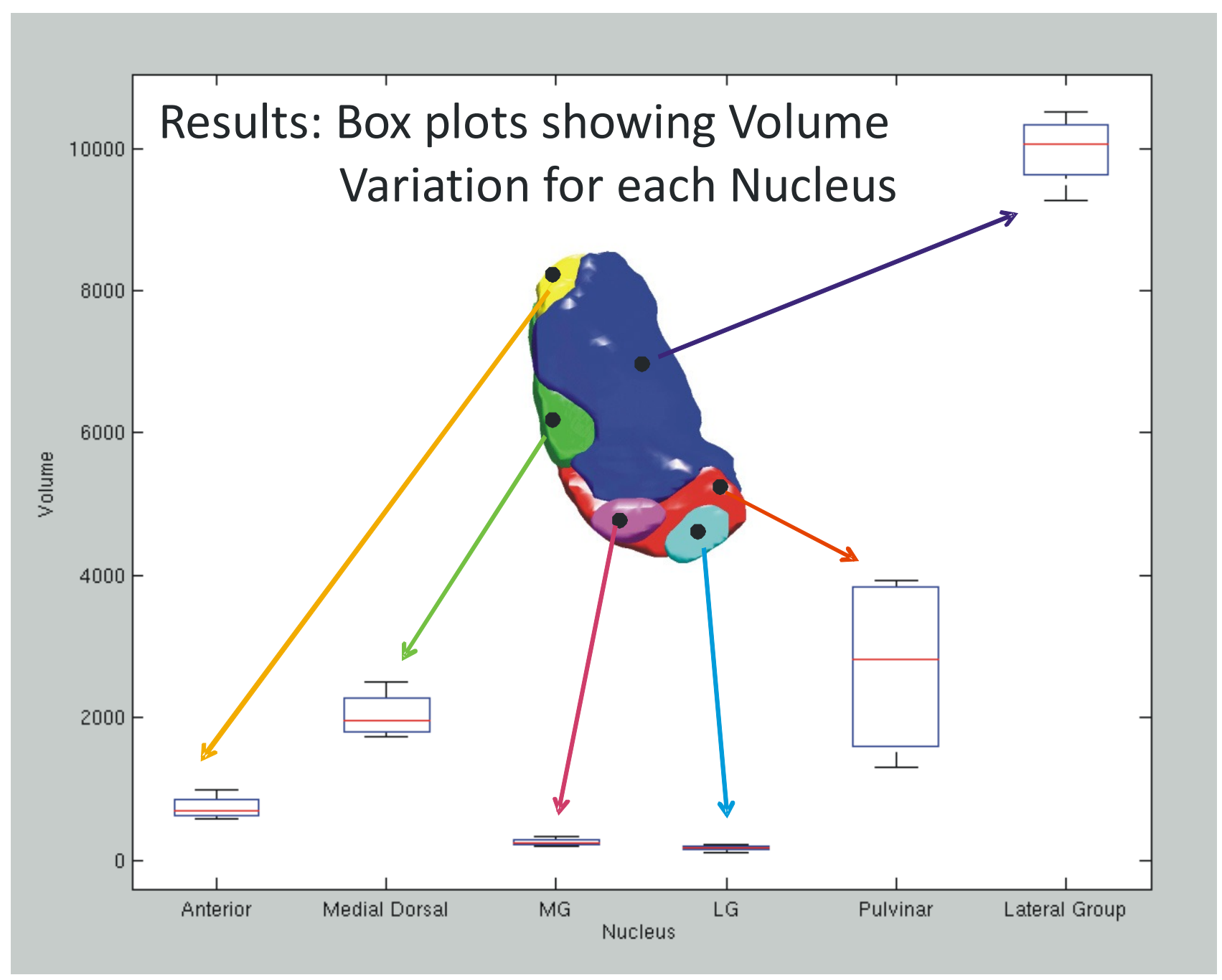

Fig. 13. Volume in cubic millimeters of different thalamic nuclei and nuclear groups evaluated by 4 raters on one subject.

\section{CONCLUSION}

We proposed a new DTI contrast - and edge map of orientation - to display variations of the principal orientation of the diffusion tensor. The edge map of the non-directional principal eigenvector is created through the 3D to 5D vector representation and the Frobenius norm of the spatial gradient matrix. The resulting image shows clearly the edges between the main nuclei in the thalamus, and agrees with qualitative information from anatomical atlases. This contrast enables us to perform reproducible manual delineations of the thalamic nuclei and will enable new measurements of this central structure of the brain as well as the quantitative validation of automated parcellation methods.

\section{ACKNOWLEDGEMENTS}

This research was supported by the following grants: NSF REU EEC0649069, NIH/NINDS 1 R01 NS056307, and NIH/NIDA K25DA025356. 


\section{REFERENCES}

[1] Mori, S., Wakana, S., Nagae-Poetscher, L.M. and van Zijl, P.C.M., [MRI atlas of human white matter], Elsevier, Amsterdam, (2005).

[2] Wiegell, M.R., Larsson, H.B.W. and Wedeen, V.J., "Fiber Crossing in Human Brain Depicted with Diffusion Tensor MR Imaging,” Radiology, 217, 897-903 (2000).

[3] Jonasson, L., Hagmann, P. and Thiran, J.-P., "A level set method for segmentation of the thalamus and its nuclei in DT-MRI,” Signal Processing, 87, 309-321 (2007).

[4] Wang, Z. and Vemuri, B.C., "DTI Segmentation Using and Information Theoretic Tensor Dissimilarity Measure," IEEE Transactions on Medical Imaging, 24(10), 1267-1277 (2005).

[5] Rieger, B. and van Vliet, L.J., "Representing Orientation in n-Dimensional Spaces," Computer Analysis of Images and Patterns, LNCS 2756, 17-24 (2003).

[6] Knutsson, H., "Producing a continuous and distance preserving 5-D vector representation of 3-D orientation," IEEE Computer Society Workshop on Computer Architecture for Pattern Analysis and Image Database Management (CAPAIDM), 175-182 (1985).

[7] Fan, X., Bazin, P.-L., Bogovic, J.A., Bai, Y. and Prince, J.L., "A multiple geometric deformable model framework for homeomorphic 3D medical image segmentation," Mathematical Methods in Biomedical Image Analysis, 23(28), 1-7 (2008).

[8] Kumar, R., Vemuri, B.C. and Mareci, T.H., "Multi-fiber Reconstruction from DW-MRI Using a Continuous Mixture of Hyperspherical von Mises-Fisher Distributions," Information Processing in Medical Imaging, 139-150 (2009)

[9] Nolte, J. and Angevine Jr., J.B., [The human brain in photographs and diagrams], Mosby, St. Louis, (1995). 